\title{
Developmental pathways from childhood conduct problems to early adult depression: findings from the ALSPAC cohort
}

\author{
Argyris Stringaris, Glyn Lewis and Barbara Maughan
}

\section{Background}

Pathways from early-life conduct problems to young adult depression remain poorly understood.

\section{Aims}

To test developmental pathways from early-life conduct problems to depression at age 18 .

\section{Method}

Data $(n=3542)$ came from the Avon Longitudinal Study of Parents and Children (ALSPAC). Previously derived conduct problem trajectories (ages 4-13 years) were used to examine associations with depression from ages 10 to 18 years, and the role of early childhood factors as potential confounders.

\section{Results}

Over $43 \%$ of young adults with depression in the ALSPAC cohort had a history of child or adolescent conduct problems, yielding a population attributable fraction of 0.15 (95\% Cl 0.08-0.22). The association between conduct problems and depression at age 18 was considerable even after adjusting for prior depression (odds ratio 1.55, 95\% Cl 1.24-1.94). Early-onset persistent conduct problems carried the highest risk for later depression. Irritability characterised depression for those with a history of conduct problems.

\section{Conclusions}

Early-life conduct problems are robustly associated with later depressive disorder and may be useful targets for early intervention.

\section{Declaration of interest}

None.
Conduct problems are common in youth, are potent risks for poor psychiatric and psychosocial outcomes in adulthood, and carry high costs to society. ${ }^{1,2}$ An important outstanding question concerns the extent to which children with conduct problems are at increased risk for adult depression. Although some studies suggest that conduct problems in youth are a precursor to adult depression, ${ }^{3-5}$ others have failed to find an association. ${ }^{6}$ Confirming the extent and nature of the link between conduct problems and early adult depression is, however, important from a clinical and public health point of view. There are effective treatments for conduct problems. ${ }^{7}$ If there were a causal relationship between depression and conduct problems, then the effectiveness of such treatments in preventing the progression to depression should also be assessed. From an aetiological perspective, understanding the mechanisms that lead children with conduct problems to become depressed would become a research priority. To examine associations between conduct problems and adult depression we use data from the Avon Longitudinal Study of Parents and Children (ALSPAC), a large population-based cohort which has a number of advantages for a study of this kind, including information about conduct problems starting early in childhood (4 years of age), data on potential risk factors, and multiple assessments from pregnancy through to young adulthood. We sought to answer five main questions:

(a) What is the risk for adult depression in children with early-life conduct problems, and how does this risk compare with that of early-life depression? Moreover, is the association between conduct problems and depression robust to adjustment for early depression?

(b) Do some subtypes of conduct problems carry a particular risk for early adult depression? We focused on age at onset as a key differentiating factor, in light of extensive research into the hypothesis that disruptive behaviours emerging in childhood differ from adolescent-onset conduct problems in both aetiology and course. ${ }^{8,9}$

(c) Do young people with a history of conduct problems show the typical adolescent rise ${ }^{10}$ in depressive symptoms?

(d) Are depressive symptom profiles similar in young people with depression with and without a history of conduct problems? We anticipated that irritability would be more common among young adults with depression and a history of conduct problems, based on the finding ${ }^{11}$ that conduct problems are more common in adolescents with depression who are also irritable.

(e) Although much past attention has focused on adolescnt risk factors for depression in teenagers with conduct problems, ${ }^{12}$ little research has focused on the role of early childhood factors. The rich early-life data available in the ALSPAC cohort allow us to examine the role of a range of potential confounders.

\section{Method}

\section{Sample}

ALSPAC is an ongoing population-based study designed to investigate the effects of a number of factors on health and development. All women resident in Avon, UK, with expected dates of delivery between 1 April 1991 and 31 December 1992 were contacted and eligible for participation. The study cohort consisted of 14541 pregnancies and 13988 children still alive at 12 months of age. Compared with the 1991 UK national census data (available at http://www.ons.gov.uk), the sample showed a slightly higher proportion of homeowners and a smaller proportion of mothers from Black and minority ethnic groups. As described by Boyd et al, ${ }^{13}$ children enrolled in ALSPAC were more educated at 16 compared with the national average, were 
more likely to be White (reflecting the ethnic composition of the area from which the sample was drawn) and less likely to be eligible for free school meals (an indicator of low income). Ethical approval for the study was obtained from the ALSPAC Law and Ethics Committee and local research ethics committees. Detailed information has been collected on the cohort since early pregnancy, including regular self-reported information from the mothers, and child assessments made in research clinics. The study website contains details of all the data that are available through a fully searchable data dictionary: www.bris.ac.uk/alspac/researchers/data-access/data-dictionary.

\section{Measures}

\section{Young adult depression}

Young adult depression was assessed using the Revised Clinical Interview Schedule (CIS-R), ${ }^{14}$ a self-administered, computerised interview completed at age 18 at the research clinic (mean age at attendance 17 years 10 months). The CIS-R establishes the severity of core symptoms of depressive disorders (depression, depressive thoughts, fatigue, sleep and concentration problems), along with diagnostic cut-points for depressive disorders, using algorithms based on ICD-10 criteria. ${ }^{15}$ Each symptom is scored on a $0-4$ scale (depressive thoughts $0-5$ ), according to the severity (frequency, duration and unpleasantness) of the symptom experienced. The CIS-R also provides indicators of the severity of irritability (scored 0-4), anhedonia (scored 1-3) and suicide risk (scored 0-3), and the frequency of self-blame (scored 1-4). A depression severity score based on the sum of the ICD-10 symptom criteria in the CIS-R (depression, depressive thoughts, sleep and concentration problems and fatigue; range 0-21, Cronbach's $\alpha=0.77$ ) was used as an adjustment in analyses of individual depression symptoms (see below).

\section{Depressive symptoms in late childhood and adolescence}

Depressive symptoms in late childhood and adolescence were assessed using the short (13-item) Mood and Feelings Questionnaire $(\mathrm{MFQ})^{16,17}$ to enquire about the occurrence of depressive symptoms over the past 2 weeks. The MFQ was completed by ALSPAC study members at research clinics at ages 10.5 and 13 years and by postal questionnaire at 16 years; slight variations in actual ages at questionnaire completion were unrelated to levels of depressive symptoms. The internal construct validity of the MFQ has been extensively supported, including in a UK community sample, which confirmed a single continuum of severity of depressive symptoms. ${ }^{18}$ The internal reliabilities (Cronbach's $\alpha$ ) of the items in the ALSPAC sample were 0.80, 0.86 and 0.91 at ages 10, 13 and 16 respectively. As expected, MFQ scores varied by gender across the three time points, with higher scores among boys at age 10, and among girls at ages 13 and 16 (age 10, boys: mean $=4.05$ (s.d. $=3.38$ ), girls: mean $=3.85$ (s.d. $=3.51), b=-0.19,95 \%$ CI -0.37 to $-0.02, \quad P<0.05$; age 13 , boys: mean $=4.07$ (s.d. $=3.80)$, girls: mean $=5.60 \quad$ (s.d. $=4.79), \quad b=1.53,95 \%$ CI 1.29 to $1.78, P<0.001$; age 16 , boys: mean $=4.26$ (s.d. $=4.57$ ), girls: mean $=6.73$ (s.d. $=5.82$ ) $, \quad b=2.47,95 \%$ CI 2.15 to 2.78 , $P<0.001)$. For the purpose of estimating population attributable fractions (PAFs; see below), total MFQ scores at ages 10 and 13 were dichotomised at $\geqslant 90$ th percentile as a 'high' cut-off.

\section{Conduct problems}

Maternal reports of child conduct problems were collected at ages $4,7,8,10,12$ and 13 years using the Strengths and Difficulties Questionnaire (SDQ), a widely-used screening instrument with well-established reliability and validity. ${ }^{19,20}$ The conduct problem subscale of the SDQ includes five items designed to assess a broad spectrum of disruptive behaviours including fighting, lying, stealing, disobedience and temper outbursts. Four developmental trajectories of conduct problems were identified in previous analyses using growth mixture models; ${ }^{21}$ an early-onset persistent class (EOP, $7.8 \%$ of the present analytic sample, $56.4 \%$ boys), who initiated conduct problems early in childhood and persisted into the early teens; a childhood-limited class (CL, 14.0\% of sample, $52.9 \%$ boys), who initiated conduct problems in early childhood but desisted to low levels by age 13; an adolescent-onset class (AO, $10.7 \%$ of sample, $51.1 \%$ boys), who started at low levels of conduct problems in childhood but increased by age 13; and a 'low' class (low, $67.5 \%$ of sample, $48.8 \%$ boys), who maintained low levels of conduct problems throughout. For some analyses, we combined the three 'high' conduct problem trajectory classes (EOP, CL and AO) to create a 'conduct problems ever' category, and contrasted this with the 'low' class.

\section{Early family, parental and sociodemographic adversity}

Multiple indicators of family, parental and sociodemographic risk were assessed during pregnancy via a Family Adversity Index (FAI). ${ }^{22}$ The FAI consisted of 17 items (including maternal age, education and marital status, housing and financial difficulties, partner relationships and support, parental psychopathology, substance use and crime) taken from questionnaires administered throughout the mother's pregnancy $(8,12,18$ and 32 weeks gestation). If adversity was present, an item was rated as 1 and scores were summed to create a scale (range 0-10). Just over half of cases $(52.6 \%)$ in the present sample were not exposed to any of these adversities, $28.2 \%$ to one, and $19.3 \%$ to two or more.

\section{Child characteristics}

Temperament was assessed using the emotionality subscale of the Emotionality, Activity, Sociability (EAS) Temperament questionnaire (score range $0-20$, mean $=7.71$, s.d. $=3.34$ ), ${ }^{23}$ completed by mothers when the study children were 69 months old. Reading was assessed using the WORD ${ }^{24}$ test of reading accuracy (score range $0-52$, mean $=28.78$, s.d. $=9.05$ ) administered by trained psychologists in research clinics at age 7.5 years. Self-esteem was assessed at age 8 at the research clinics using the scholastic competence subscale of Harter's Self-Perception Profile for Children $^{25}$ (score range 6-24, mean $=17.09$, s.d. $=3.62$ ).

\section{Attrition}

Data to estimate conduct problem trajectories were available for 7218 young people. As detailed in past reports, ${ }^{21}$ cases with missing data for trajectory assignments differed from the full birth cohort on a number of sociodemographic background factors (including higher rates of teen motherhood and maternal smoking in pregnancy, and lower levels of maternal education). Age 18 depression data were available for 3542 individuals with conduct problem trajectory assignments. Girls were more likely than boys to participate at age $18\left(54.7 \%\right.$ v. $43.5 \%$; Pearson $\chi^{2}(1)=90.25$; $P<0.001)$. Adjusted for gender, trajectory classes were also significant predictors of participation at age 18, with odds ratios (ORs) ranging from 0.83 (95\% CI $0.76-0.91, P<0.001$ ) for those in the CL class to 0.69 (95\% CI $0.60-0.80, P<0.001)$ for those in the EOP class. To ensure the robustness of our findings we repeated the study's main analyses using inverse probability weighting (see online supplement for details) and found essentially the same pattern of results; we therefore present the unweighted, full case analyses in this paper. 


\section{Statistical methods}

\section{conduct problem trajectories and young adult depression}

We estimated associations between age 18 depression and either conduct problems ever or conduct problem trajectory classes using logistic regression models, with age 18 depression diagnosis as outcome and conduct problems ever or trajectory classes as predictors. To compare the estimates obtained from the various conduct problem indicators with those for depression, the dichotomised MFQ scores were entered either on their own or together with conduct problems ever or conduct problem trajectory classes in logistic regression models. The PAF for the predictors was estimated in Stata version 11.2 for Windows using the punaf command. Unless otherwise indicated, the low conduct problem trajectory class was used as the reference category. We present odds ratios, 95\% confidence intervals and $P$-values from these analyses.

\section{Conduct problems and the development of depressive symptoms in adolescence}

The relationship between conduct problem trajectories and MFQ scores at age 10 years was examined in a linear regression model, with trajectories as predictors; we present $b$-coefficients with $95 \%$ confidence intervals and $P$-values. To examine associations between trajectories and the development of depressive symptoms across adolescence, we estimated the baseline levels ('intercept' at age 10) and rate of change ('slope' between ages 10 and 16) of MFQ scores in Mplus. ${ }^{26}$ We imported these estimates into Stata and used them in linear regression models with the slope as the outcome and trajectory classes as the predictors, adjusting for baseline (age 10) level of depression; we present regression coefficients with $95 \%$ confidence intervals and $P$-values.

\section{Depressive symptom profiles}

Associations between trajectory classes and depression symptoms at age 18 were estimated in ordinal logistic regression models with symptom scores as outcomes, adjusted for the overall depression severity score based on the sum of the ICD-10 symptom criteria in the CIS-R. We present odds ratios, $95 \%$ confidence intervals and $P$-values from these analyses.

\section{Early childhood confounds}

These were entered in logistic regression models with age 18 depression diagnosis as outcome and conduct problems ever and conduct problem trajectory classes as predictors. We present odds ratios, 95\% confidence intervals and $P$-values from these analyses.

Unless otherwise specified, all analyses were undertaken in Stata version 11.2 for Windows, with cases weighted by the posterior probabilities of trajectory group membership throughout.

\section{Results}

\section{Childhood conduct problems and young adult depression}

There was a strong association between young adult depression and both conduct problems ever and the conduct problem trajectory classes, as illustrated in Fig. 1. Overall, $42.3 \%$ of those with depression at age 18 had shown high levels of conduct problems at some point in childhood or early adolescence. As expected, girls were more likely to meet depression criteria than boys $(\mathrm{OR}=2.54,95 \% \mathrm{CI} 1.98-3.27, P<0.001)$. Adjusted for these strong gender effects, both conduct problems ever and conduct problem trajectory classes remained significantly associated with risk for depression at age 18 (Table 1). There was no evidence of a gender $\times$ trajectory class interaction $(F(3,6058)=0.47$, $P=0.70$ ). In addition, young people with early-onset and persistent conduct problems were at significantly higher risk of depression at age 18 than those in the $\mathrm{CL}(\mathrm{OR}=0.58,95 \% \mathrm{CI}$ $0.40-0.82, P=0.002)$ and $\mathrm{AO}(\mathrm{OR}=0.65,95 \%$ CI $0.46-0.92$, $P=0.014)$ classes.

We next compared the following in terms of their association with young adult depression: (a) conduct problems ever or conduct problem trajectory classes; (b) depression in late childhood or early adolescence (age 10 and 13 MFQ scores). Table 1 presents the odds ratios for the association between each predictor and outcome adjusted for gender, and also the PAFs for the predictors of interest. The PAF estimate for adult depression due to conduct problems ever was about twice as large as that for depression at age 10 years, and comparable to that for depression at age 13 years (15\% v. 19\%). Finally, we estimated the PAF due to conduct problems adjusting for high depression scores at ages 10 and 13 years. As can be seen in Table 1, the PAF estimates changed only slightly (from 0.15 to 0.14 ) following the adjustment.

\section{Conduct problem trajectories and the development of depressive symptoms in adolescence}

Figure 2 illustrates the development of depressive symptoms (as assessed by mean total MFQ scores) in each gender and trajectory class from late childhood to middle adolescence. At age 10 years there was a significant effect of trajectory class, with young people in each of the 'conduct problem' trajectories showing significantly higher symptom levels than those in the low class (CL: $b=0.61$, $95 \%$ CI 0.42 to 0.81 ; $\mathrm{AO}: b=0.84,95 \%$ CI 0.64 to 1.05 ; EOP: $b=1.62,95 \%$ CI 1.30 to 1.95 ; all $P<0.001)$. There was no significant gender $\times$ conduct problem trajectory class interaction $(F(3,6709)=$ $2.29, P=0.08$ ). We next estimated the intercept (mean for boys $=3.84$ s.d. $=1.22$; mean for girls $=4.07$, s.d. $=1.34$ ) and slope (mean for boys 0.70 , s.d. $=0.70$; mean for girls 0.91 , s.d. $=0.88$ ) for depressive symptoms between ages 10,13 and 16 years. As expected, there was a considerable increase in depressive symptom levels between ages 10 and 16 years (Fig. 2), reflected in the results from a linear growth model (estimate for slope $=0.90,95 \%$ CI 0.82 to 0.98 , $P<0.001)$. In a model estimating the rate of change (slope) in depressive symptoms across ages 10, 13 and 16 years, gender was a significant predictor, with girls showing stronger increases in symptom scores than boys $(b=0.30,95 \%$ CI 0.26 to 0.33 , $P<0.001)$. As Fig. 2 suggests, however, there was no difference in the rate of change in depressive symptoms by conduct problem trajectory after gender and baseline (age 10) symptom levels

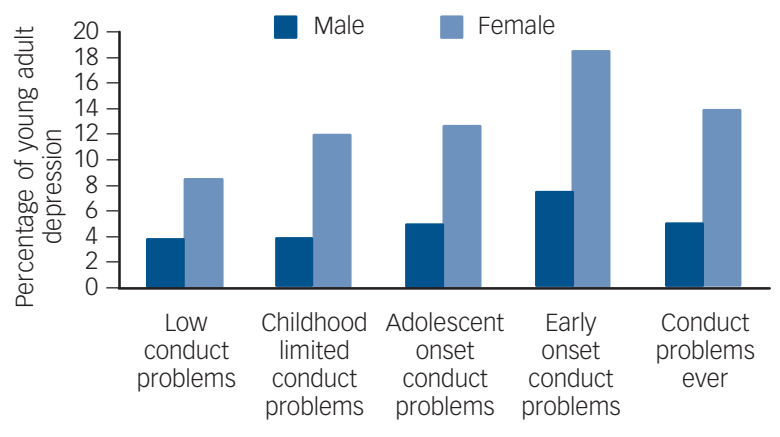

Fig. 1 Young adult depression by conduct problem trajectories and gender. 
Table 1 Predictors of young adult depression by conduct problems ever, conduct problem trajectories and early depression

\begin{tabular}{|c|c|c|c|c|c|c|c|}
\hline & \multirow{2}{*}{$\begin{array}{l}\text { Conduct } \\
\text { problems ever }\end{array}$} & \multicolumn{3}{|c|}{ Conduct problem trajectory class } & \multicolumn{3}{|c|}{$\begin{array}{l}\text { Late childhood/early adolescent depression } \\
\quad(\geqslant 90 \text { th percentile MFQ score })\end{array}$} \\
\hline & & $\mathrm{CL}$ & $\mathrm{AO}$ & EOP & Age 10 & Age 13 & Age 10 or 13 \\
\hline \multicolumn{8}{|c|}{ Adjusted for gender } \\
\hline OR & 1.62 & 1.34 & 1.49 & 2.32 & 1.89 & 3.28 & 2.73 \\
\hline $\begin{array}{l}(95 \% \mathrm{Cl}) \\
\text { PAF }\end{array}$ & $(1.30-2.01)$ & (1.03-1.73) & (1.16-1.94) & (1.64-3.27) & $\begin{array}{c}(1.30-2.75) \\
0.06\end{array}$ & $\begin{array}{c}(2.40-4.48) \\
-19\end{array}$ & (2.10-3.60) \\
\hline $\begin{array}{l}\text { PAF } \\
(95 \% \mathrm{Cl})\end{array}$ & $\begin{array}{c}0.15 \\
(0.08-0.22)\end{array}$ & $\begin{array}{c}0.04 \\
(0.00-0.07)\end{array}$ & $\begin{array}{c}0.04 \\
(0.01-0.07)\end{array}$ & $(0.04-0.11)$ & $\begin{array}{c}0.06 \\
(0.02-0.11)\end{array}$ & $\begin{array}{c}0.19 \\
(0.12-0.25)\end{array}$ & $(0.14-0.27)$ \\
\hline \multicolumn{8}{|c|}{$\begin{array}{l}\text { Adjusted for gender } \\
\text { and depression age } 10\end{array}$} \\
\hline $\begin{array}{l}\mathrm{OR} \\
(95 \% \mathrm{Cl})\end{array}$ & $\begin{array}{c}1.64 \\
(1.31-2.06)\end{array}$ & $\begin{array}{c}1.39 \\
(1.06-1.81)\end{array}$ & $\begin{array}{c}1.46 \\
(1.11-1.92)\end{array}$ & $\begin{array}{c}2.39 \\
(1.69-3.39)\end{array}$ & - & - & - \\
\hline PAF & 0.16 & 0.04 & 0.04 & 0.08 & & & \\
\hline$(95 \% \mathrm{Cl})$ & $(0.08-0.23)$ & $(0.00-0.07)$ & $(0.00-0.07)$ & $(0.04-0.11)$ & & & \\
\hline \multicolumn{8}{|c|}{$\begin{array}{l}\text { Adjusted for gender } \\
\text { and depression age } 13\end{array}$} \\
\hline $\begin{array}{l}\text { OR } \\
(95 \% \text { Cl) }\end{array}$ & $\begin{array}{c}1.63 \\
(1.29-2.05)\end{array}$ & $\begin{array}{c}1.41 \\
(1.08-1.86)\end{array}$ & $\begin{array}{c}1.51 \\
(1.13-2.01)\end{array}$ & $\begin{array}{c}2.19 \\
(1.50-3.19)\end{array}$ & - & - & - \\
\hline PAF & 0.15 & 0.04 & 0.04 & 0.07 & & & \\
\hline$(95 \% \mathrm{Cl})$ & $(0.07-0.22)$ & $(0.01-0.08)$ & $(0.01-0.07)$ & $(0.03-0.10)$ & & & \\
\hline \multicolumn{8}{|c|}{$\begin{array}{l}\text { Adjusted for gender and } \\
\text { depression age } 10 \text { or } 13\end{array}$} \\
\hline $\begin{array}{l}\text { OR } \\
(95 \% \mathrm{Cl})\end{array}$ & 1.55 & 1.35 & & 2.19 & - & - & - \\
\hline $\begin{array}{l}(75 \% \text { Cl) } \\
\text { PAF }\end{array}$ & $\begin{array}{c}(1.24-1.94) \\
0.14\end{array}$ & $\begin{array}{c}(1.03-1.16) \\
0.04\end{array}$ & $\begin{array}{c}(1.04-1.81) \\
0.03\end{array}$ & $\begin{array}{c}(1.54-3.11) \\
0.07\end{array}$ & & & \\
\hline$(95 \% \mathrm{Cl})$ & $(0.06-0.21)$ & $(0.00-0.07)$ & $(0.00-0.06)$ & $(0.03-0.11)$ & & & \\
\hline
\end{tabular}

had been taken into account (CL: $b=0.01,95 \% \mathrm{CI}-0.02$ to 0.05 ; AO: $b=0.03,95 \% \mathrm{CI}-0.01$ to 0.07 ; EOP: $b=0.02,95 \% \mathrm{CI}-0.04$ to 0.07 ; all $P>0.15$ ). There was also no significant gender $\times$ conduct problem trajectory interaction in the prediction of the rate of symptom change $(F(3,6939)=0.51, P=0.67)$.

\section{Depressive symptom profiles}

Figure 3 focuses on young people who met criteria for depression at age 18, and shows mean symptom severity scores for each conduct problem trajectory class. Preliminary tests showed no

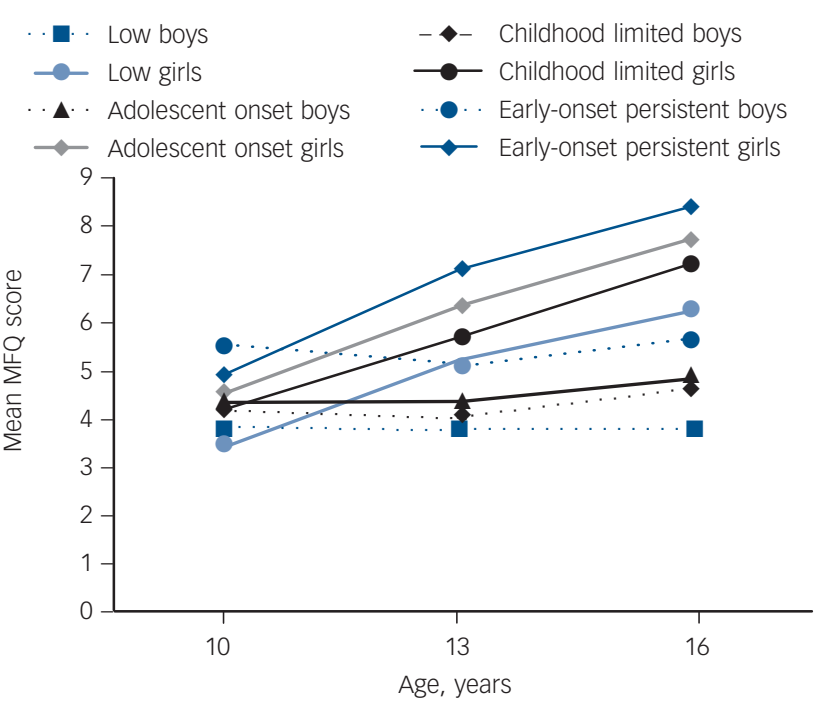

Fig. 2 Mean Mood and Feelings Questionnaire (MFQ) scores at ages 10, 13 and 16 years by conduct problem trajectories and gender. evidence of gender differences in symptom severity within individuals meeting criteria for depression $(b=0.59,95 \%$ CI -0.34 to $1.51, P=0.214$ ), so data for males and females are combined. In general, as Fig. 3 suggests, depressive symptom profiles were very similar irrespective of conduct problem history. In keeping with our hypothesis, however, irritability was significantly more common in the EOP $(\mathrm{OR}=2.40,95 \%$ CI 1.34 to $4.31, P=0.004)$ and $\mathrm{CL}(\mathrm{OR}=1.59,95 \% \mathrm{CI} 1.03$ to 2.45 , $P=0.04)$ classes than in the low class; young people classified as showing adolescent-onset conduct problems did not differ significantly from the low group in levels of irritability $(\mathrm{OR}=1.29,95 \% \mathrm{CI} 0.77$ to $2.16, P=0.33)$. After adjusting for overall depression severity, irritability was still significantly more common in the EOP $(\mathrm{OR}=2.16,95 \%$ CI 1.24 to 3.78 , $P=0.007)$ than in the low class; adjustment for depression severity

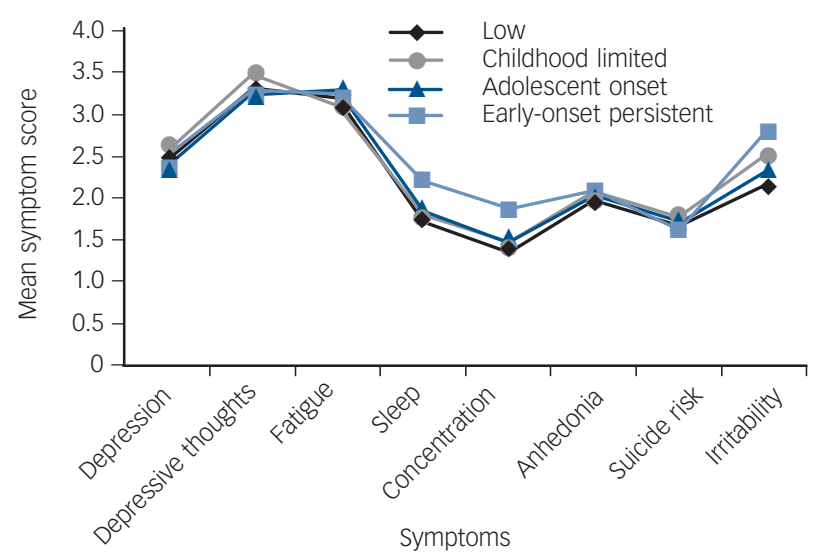

Fig. 3 Mean age 18 depression symptom scores by conduct problem trajectories: individuals meeting criteria for depression only. 


\begin{tabular}{|c|c|c|c|c|}
\hline & \multirow{2}{*}{$\begin{array}{l}\text { Conduct problems ever } \\
\text { Adjusted OR }{ }^{a} \\
(95 \% \mathrm{Cl})\end{array}$} & \multicolumn{3}{|c|}{ Conduct problem trajectory class } \\
\hline & & $\begin{array}{l}\text { CL Adjusted OR } \\
\qquad(95 \% \mathrm{Cl})\end{array}$ & $\begin{array}{l}\text { AO Adjusted } \mathrm{OR}^{\mathrm{a}} \\
\qquad(95 \% \mathrm{Cl})\end{array}$ & $\begin{array}{l}\text { EOP Adjusted } \mathrm{OR}^{\mathrm{a}} \\
\qquad(95 \% \mathrm{Cl})\end{array}$ \\
\hline $\begin{array}{l}\text { Baseline model (reference category for } \\
\text { trajectory class comparisons = 'low') }\end{array}$ & $1.62(1.30-2.01)$ & $1.34(1.03-1.73)$ & $1.50(1.16-1.94)$ & $2.32(1.64-3.27)$ \\
\hline \multicolumn{5}{|l|}{ Adjusted for: } \\
\hline Family Adversity Index & $1.48(1.19-1.83)$ & $1.27(0.98-1.64)$ & $1.40(1.08-1.81)$ & $1.99(1.40-2.82)$ \\
\hline \multicolumn{5}{|l|}{ Child characteristics } \\
\hline Temperament (emotionality, age 5 years) & $1.53(1.23-1.90)$ & $1.29(0.99-1.68)$ & $1.42(1.08-1.86)$ & $2.21(1.55-3.14)$ \\
\hline Reading (age 7 years) & $1.69(1.36-2.11)$ & $1.44(1.10-1.87)$ & $1.52(1.17-1.99)$ & $2.51(1.75-3.60)$ \\
\hline Academic self-esteem (age 8 years) & $1.55(1.23-1.95)$ & $1.28(0.97-1.69)$ & $1.42(1.07-1.87)$ & $2.29(1.59-3.31)$ \\
\hline All significant confounders ${ }^{b}$ & $1.50(1.18-1.89)$ & $1.30(0.98-1.71)$ & $1.40(1.06-1.86)$ & $2.08(1.40-3.08)$ \\
\hline
\end{tabular}

led to a minimal reduction of the odds ratio for the CL $(\mathrm{OR}=1.54,95 \%$ CI 0.98 to 2.43$)$ compared with the low class, although it rendered the association non-significant $(P=0.063)$. We also found that youth with depression in the EOP class (but not in the CL or AO classes) reported significantly higher levels of sleep $(\mathrm{OR}=1.95,95 \% \mathrm{CI} 1.08$ to $3.51, P=0.026)$ and concentration $(\mathrm{OR}=2.04,95 \% \quad \mathrm{CI} 1.12$ to $3.72, \quad P=0.02)$ difficulties than those in the low class. However, both these associations were reduced after adjusting for depression severity (sleep: $\mathrm{OR}=1.87,95 \% \mathrm{CI} 0.92$ to $3.80, P=0.082$; concentration: $\mathrm{OR}=1.73,95 \%$ CI 0.91 to $3.27, P=0.094$ ).

\section{Early childhood confounds}

Finally, we explored the extent to which early childhood characteristics and exposures contributed to the increased risk of early adult depression among young people with a history of conduct problems. As Table 2 shows, adjustments for early family adversity, childhood temperament and self-esteem each resulted in modest reductions in the odds ratios for conduct problems, whereas adjustments for age 7 reading skills resulted in slight increases. In a multivariate model early adversity, reading level and academic self-esteem each retained independent associations with age 18 depression. Joint adjustment for all of these confounders resulted in a $19 \%$ reduction in the odds ratio for conduct problems ever, and reductions of $12 \%(\mathrm{CL}), 20 \%(\mathrm{AO})$ and $18 \%(\mathrm{EOP})$ in the odds ratios for the three 'high' conduct problem trajectory classes.

\section{Discussion}

In this paper we set out to address five key outstanding questions concerning the extent and nature of the overlap between conduct problems in childhood and early adult depression.

The first was to address current ambiguities in the relationship between conduct problems in youth as a precursor of adult depression, with some studies suggesting an association, ${ }^{3-5}$ but others failing to find a link. ${ }^{6}$ It is probably as a result of this uncertainty that, despite increasing interest in early antisocial behaviours, depression is only rarely addressed as one of its outcomes. ${ }^{7}$ In our study using a large birth cohort, we confirm that conduct problems were strong markers of risk for subsequent depression: over $43 \%$ of young adults with depression in the ALSPAC cohort had a history of conduct problems in childhood or adolescence. Our study also demonstrates the potential public health importance of these findings by showing that PAFs for adult depression due to conduct problems were comparable to those due to early adolescent depression. This would imply that a considerable number of cases of young adults with depression could be prevented if early conduct problems were treated adequately and the association were causal. Effective treatments for conduct problems in youth ${ }^{7}$ might prevent some future depression, a hypothesis that our findings suggest should be tested empirically. Moreover, our study shows that the prediction from early conduct problems to young adult depression is largely independent of depression at ages 10 and 13 years. Of note, the prediction from conduct problems to young adult depression is cross-informant (from early parent- to later self-report), adding to the robustness of our findings.

Against this background, we turned to our second question: whether particular subtypes of conduct problems might carry an especially high depression risk. We focused on variations in age at onset, in light of extensive research on their roles as key markers of heterogeneity in antisocial behaviour. ${ }^{8}$ More recently, distinctions have also been drawn within early-onset cases between individuals that persist in antisocial activities and those that do not. ${ }^{9}$ To date, however, few studies have examined variations among these subtypes in risk for early adult depression. One possibility is that early-onset conduct problems (which typically show poor later outcomes) would also carry the strongest risk for depression; another is that conduct problems may be more strongly associated with depression if they are present in adolescence, when depressive symptoms themselves show a sharp increase. ${ }^{27}$

Of the few tests of these possibilities reported thus far, Odgers et al, ${ }^{9}$ using data from the Dunedin longitudinal cohort, provide the closest match to our characterisation of child and adolescent conduct problems. They examined past-year rates of major depressive disorder in adulthood (age 32) for individuals following child and adolescent antisocial trajectories broadly similar to those examined here. In both men and women, risks of depression in the early 30s were markedly elevated for those on a 'life course persistent' antisocial trajectory, but were not significantly elevated for those on either childhood- or adolescence-limited pathways. Our findings were consistent with this pattern in finding much elevated rates of depression among young people with early-onset and persistent conduct problems, significantly higher than those in all other conduct problem trajectory groups. In addition, however, our findings suggested elevated risks for young people with both childhood-limited and adolescent-onset conduct problems. It is unclear at this stage how far these variations reflect differences in the ages at which 
depression was assessed (age 18 in ALSPAC, age 32 in Dunedin) or in other methodological factors. What is clear from both studies, however, is that conduct problems that begin early in childhood and persist into adolescence carry a particularly potent risk for subsequent depression.

Our third question concerned the development of depressive symptoms earlier in adolescence, and the extent to which young people with histories of conduct problems showed the typical profile of rising depression levels in the early to mid teens now widely established in population samples. ${ }^{27}$ We found that children with conduct problems had significantly higher depression scores than their counterparts without conduct problems from late childhood onwards, but that the rate at which depression symptoms rose over the following years was similar in all groups. If replicated, these findings suggest that links between conduct problems and depression are established early and therefore through mechanisms that are independent of adolescent processes.

We tested for possible gender differences in depression risk, prompted by the 'gender paradox' hypothesis, ${ }^{28}$ whereby girls with conduct problems are thought to show higher rates of comorbidity with depression than boys, despite lower overall rates of conduct problems. Consistent with past studies, we found that early adult depression was more common in females than males. However, we found no evidence that the rates of comorbidity between depression and conduct problems were higher in girls than boys. Importantly, these findings applied not only to early adult depression, but also to rising symptom levels in adolescence: the rise in symptoms of depression was stronger in girls (as indicated by the statistical estimates of the slope), but this effect was not moderated by conduct problem trajectory. Previous findings have provided mixed support for the gender paradox. ${ }^{29}$ We provide a relatively rigorous test of this hypothesis here, based on a cross-informant approach (parent-report for conduct problems and self-report for depression), and using two different instruments to ascertain depression at different developmental stages.

Our fourth question concerned the pattern of depressive symptoms across trajectories. Among young adults who met criteria for depression, the profile of depressive symptoms was generally similar irrespective of conduct problem history. We had predicted that irritability might differentiate young people with depression with conduct problem histories from those without, and found some support for this hypothesis, in that young people in the EOP group had significantly higher irritability scores compared with those in the low conduct problems class, controlling for depression severity. In a previous cross-sectional epidemiological study of adults, ${ }^{30}$ irritability was present in about half of respondents with lifetime DSM-IV major depressive disorder, and the presence of irritability was associated with earlier age at onset, higher comorbidity rates and increased disability. In addition, we have recently shown that among adolescents with depression, those with symptoms of episodic irritability were more likely to have comorbid disruptive disorder diagnoses. ${ }^{11}$ In the current sample, levels of sleep and concentration problems also showed some differences between conduct problem trajectory classes. These were non-hypothesised findings, and thus require replication in future studies.

Our final question concerned the role of early family and child characteristics in the overlap between the two disorders that we observed. In the main, past studies of conduct problem/depression overlaps have focused on adolescent and later life-course risks that might be implicated in heterotypic continuities. ${ }^{30}$ We sought to extend these findings by focusing on factors evident earlier in development. We found that adjustment for early shared family adversities, child temperament, early cognitive skills and self- esteem resulted in only modest reductions in estimates of risk for young adult depression associated with childhood conduct problems. Our study cannot determine whether these associations reflect a direct causal impact of conduct problems on risk for depression or the operation of other shared risk factors, common to both disorders. It has been argued, for example, that the adverse consequences of conduct problems for children's family and peer relationships, and for their skill development and academic performance result in a 'failure trajectory' that increases vulnerability to depression. ${ }^{31}$ In addition, however, subsequent research suggests that the relationship between depression and conduct problems can largely be accounted for by shared genetic factors. ${ }^{32,33}$ Further research on these differing pathways would clearly be of importance.

\section{Strengths and limitations}

Our study has several strengths, including a large sample size, a longitudinal design with rich measures spanning childhood to young adulthood, and data from multiple informants. As is common in longitudinal studies, however, there was loss to follow-up over the period covered by our analyses, and it was clear that these losses were selective: more socially disadvantaged individuals were excluded from the trajectory group assignments, and conduct problems were themselves significant predictors of study member participation in early adulthood. As a result, we are likely to have underestimated the prevalence of conduct problems in our analytic sample. Past evidence suggests, however, that it is much less likely that loss to follow-up would affect estimates of the associations between variables, ${ }^{34}$ and the use of inverse probability weights produced essentially similar results in the present analyses; as a result, we think it unlikely that the associations we observed between conduct problem trajectories and depression are explained by sample attrition.

\section{Clinical implications}

Our findings have a number of implications. First, in keeping with past findings, the data from this study suggest that conduct problem trajectories are a marker of risk for depression problems throughout adolescence and early adulthood; hence, clinicians treating conduct problems in children and adolescents should be alert to recognise symptoms of depression and not to miss early treatment opportunities. Second, our findings suggest that a history of childhood or adolescent conduct problems, and not solely the persistence of such problems, carries risk for the development of depression. From a prevention and treatment perspective, it is important to note that even those in the CL group - i.e. those children whose conduct problems had desisted by adolescence - were more likely than the rest of the population to have depression in early adulthood. These findings may suggest that interventions aimed at depression, over and above those aimed at targeting disruptive behaviour problems, may be necessary in children presenting with conduct problems. Future studies investigating the mechanisms underlying these transitions, and how these could inform early interventions, would be of value.

Argyris Stringaris, MD, PhD, MRCPsych, Department of Child and Adolescent Psychiatry, King's College London, Institute of Psychiatry, London; Glyn Lewis, PhD, Division of Psychiatry, University College London; Barbara Maughan, PhD, MRC Social, Genetic and Developmental Psychiatry Centre, King's College London, Institute of Psychiatry, London, UK

Correspondence: Argyris Stringaris, King's College London, Institute of Psychiatry, De Crespigny Park, London SE5 8AF, UK. Email: argyris.stringaris@kcl.ac.uk

First received 24 Jun 2013, final revision 27 Nov 2013, accepted 27 Jan 2014 


\section{Funding}

This research was specifically funded by the UK Medical Research Council (Grant ref G0500953, BM) and the Wellcome Trust (Grant ref: WT084268MA, GL). A.S. gratefully acknowledges the support of the Wellcome Trust.

\section{Acknowledgements}

We are extremely grateful to all the families who took part in this study, the midwives fo their help in recruiting them, and the whole ALSPAC team, which includes interviewers, computer and laboratory technicians, clerical workers, research scientists, volunteers, managers, receptionists and nurses. The UK Medical Research council and the Wellcome Trust (Grant ref: 092731) and the University of Bristol provide core support for ALSPAC.

\section{References}

1 Scott S, Knapp M, Henderson J, Maughan B. Financial cost of social exclusion: follow up study of antisocial children into adulthood. BMJ 2001; 323: 191

2 Kretschmer T, Hickman M, Doerner R, Emond A, Lewis G, Macleod J, et al. Outcomes of childhood conduct problem trajectories in early adulthood: findings from the ALSPAC study. Eur Child Adolesc Psychiatry 2013; Nov 7 epub ahead of print

3 Copeland WE, Shanahan L, Costello EJ, Angold A. Childhood and adolescent psychiatric disorders as predictors of young adult disorders. Arch Gen Psychiatry 2009; 66: 764-72.

4 Fergusson DM, Horwood L, Ridder EM. Show me the child at seven: the consequences of conduct problems in childhood for psychosocial functioning in adulthood. J Child Psychol Psychiatry 2005; 46: 837-49.

5 Kim-Cohen J, Caspi A, Moffitt TE, Harrington H, Milne BJ, Poulton R. Prior juvenile diagnoses in adults with mental disorder: developmental follow-back of a prospective-longitudinal cohort. Arch Gen Psychiatry 2003; 60: 709-17.

6 Althoff RR, Verhulst FC, Rettew DC, Hudziak JJ, van der Ende J. Adult outcomes of childhood dysregulation: a 14-year follow-up study. J Am Acad Child Adolesc Psychiatry 2010; 49: 1105-16.

7 Pilling S, Gould N, Whittington C, Taylor C, Scott S. Recognition, intervention, and management of antisocial behaviour and conduct disorders in children and young people: summary of NICE-SCIE guidance. BMJ 2013; 346: f1298.

8 Moffitt TE. Adolescence-limited and life-course-persistent antisocial behavior a developmental taxonomy. Psychol Rev 1993; 100: 674.

9 Odgers CL, Moffitt TE, Broadbent JM, Dickson N, Hancox RJ, Harrington H, et al. Female and male antisocial trajectories: from childhood origins to adult outcomes. Dev Psychopathol 2008; 20: 673-716.

10 Angold A, Erkanli A, Silberg J, Eaves L, Costello EJ. Depression scale scores in 8-17-year-olds: effects of age and gender. J Child Psychol Psychiatry 2002; 43: 1052-63.

11 Stringaris A, Maughan B, Copeland WS, Costello EJ, Angold A. Irritable mood as a symptom of depression in youth: prevalence, developmental and clinical correlates in the Great Smoky Mountains Study. J Am Acad Child Adolesc Psychiatry 2013; 52: 831-40.

12 Rutter M, Bishop DVM, Pine DS, Scott S, Stevenson J, Taylor E, et al (eds) Rutter's Child and Adolescent Psychiatry, Fifth Edition. Blackwell Publishing, 2009.

13 Boyd A, Golding J, Macleod J, Lawlor DA, Fraser A, Henderson J, et al. Cohort profile: the 'children of the $90 \mathrm{~s}^{\prime}$ - the index offspring of the Avon Longitudinal Study of Parents and Children. Int J Epidemiol 2013; 42: 111-27.

14 Lewis G, Pelosi AJ, Araya R, Dunn G. Measuring psychiatric disorder in the community: a standardized assessment for use by lay interviewers. Psychol Med 1992; 22: 465-86.
15 World Health Organization. The ICD-10 Classification of Mental and Behavioural Disorders: Clinical Descriptions and Diagnostic Guidelines. WHO, 1992.

16 Messer SC, Angold A, Costello EJ. Development of a short questionnaire for use in epidemiological studies of depression in children and adolescents: factor composition and structure across devlopment. Int J Methods Psychiatr Res 1995; 5: 251-62.

17 Angold A, Costello EJ, Messer SC, Pickles A, Winder F, Silver, D. The development of the short questionnaire for use in epidemiological studies of depression in children and adolescents. Int J Methods Psychiatr Res 1995; 5 $1-12$.

18 Sharp C, Goodyer IM, Croudace TJ. The Short Mood and Feelings Questionnaire (SMFQ): a unidimensional item response theory and categorical data factor analysis of self-report ratings from a community sample of 7-through 11-year-old children. J Abnorm Child Psychol 2006; 34: 379-91.

19 Goodman R. The Strengths and Difficulties Questionnaire: a research note. J Child Psychol Psychiatry 1997; 38: 581.

20 Goodman R. Psychometric properties of the strengths and difficulties questionnaire. J Am Acad Child Adolesc Psychiatry 2001; 40: 1337.

21 Barker ED, Maughan B. Differentiating early-onset persistent versus childhood-limited conduct problem youth. Am J Psychiatry 2009; 166 900-8.

22 Bowen E, Heron J, Waylen A, Wolke D. Domestic violence risk during and after pregnancy: findings from a British longitudinal study. BJOG 2005; 112: 1083-9.

23 Buss AH, Plomin R. Temperament: Early Developing Personality Traits. Lawrence Erlbaum, 1984.

24 Rust J, Golombok S, Trickey G. WORD, Wechsler Objective Reading Dimensions Manual. Psychological Corporation, 1993.

25 Harter S. Manual for the Self-Perception Profile for Children. University of Denver, 1985.

26 Muthen LK, Muthen B.O. Mplus User's Guide (5th edn). Muthen \& Muthen, 2007.

27 Thapar A, Collishaw S, Pine DS, Thapar AK. Depression in adolescence. Lancet 2012; 379: 1056-67.

28 Loeber R, Keenan, K. Interaction between conduct disorder and its comorbid conditions: effects of age and gender. Clin Psychol Rev 1994; 14: 497-523.

29 Diamantopoulou S, Verhulst FC, van der Ende J. Gender differences in the development and adult outcome of co-occurring depression and delinquency in adolescence. J Abnorm Psychol 2011; 120: 644-55.

30 Fava M, Hwang I, Rush AJ, Sampson N, Walters EE, Kessler RC. The importance of irritability as a symptom of major depressive disorder: results from the National Comorbidity Survey Replication. Mol Psychiatry 2010; 15 856-67

31 Capaldi DM. Co-occurrence of conduct problems and depressive symptoms in early adolescent boys: II. A 2-year follow-up at Grade 8. Dev Psychopathol 1992; 4: 125-44.

32 Stringaris A, Zavos $\mathrm{H}$, Leibenluft E, Maughan B, Eley TC. Adolescent irritability: phenotypic associations and genetic links with depressed mood. Am J Psychiatry 2012; 169: 47-54.

33 Tackett JL, Waldman ID, Van Hulle CA, Lahey BB. Shared genetic influences on negative emotionality and major depression/conduct disorder comorbidity. J Am Acad Child Adolesc Psychiatry 2011; 50: 818-27.

34 Wolke D, Waylen A, Samara M, Steer C, Goodman R, Ford T, et al. Selective drop-out in longitudinal studies and non-biased prediction of behaviour disorders. Br J Psychiatry 2009; 195: 249-56.

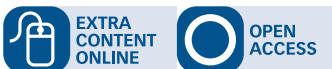

